\title{
Some geometric properties of generalized modular sequence space derived by the generalized de la Vallée-Poussin mean
}

\author{
Abdul Latif ${ }^{*}$, Chirasak Mongkolkeha ${ }^{2}$ and Wutiphol Sintunavarat ${ }^{3}$
}

"Correspondence: alatif@kau.edu.sa 'Department of Mathematics, King Abdulaziz University, P.O. Box 80203, Jeddah, 21589, Saudi Arabia Full list of author information is available at the end of the article

\begin{abstract}
In this paper, we define a generalized modular sequence space by using the generalized de la Vallée-Poussin mean with a generalized Riesz transformation. Moreover, we investigate the property $(\beta)$ and the uniform Opial property which is equipped with the Luxemburg norm. Finally, we show that this space has the fixed point property.
\end{abstract}

Keywords: generalized Cesàro sequence spaces; property $(\beta)$; uniform Opial property; Vallée-Poussin; generalized Riesz transformations; fixed point property

\section{Introduction}

A number of mathematicians are studying the geometric properties of Banach spaces, because such properties were identified as an important characteristic of the Banach spaces. For example, if Banach spaces have some geometric properties such as the uniformly rotund, $P$-convexity, $Q$-convexity, Banach-Saks property, then they are reflexive spaces. The investigations of the metric geometry of Banach spaces date back to 1913, when Radon [1] introduced the Kadec-Klee property (sometimes called the Radon-Riesz property, or property $(H)$ ), and later, when Riesz [2,3] showed that the classical $L_{p}$-spaces, $1<p<\infty$, have the Kadec-Klee property. Although the space $L_{1}[0,1]$ (with Lebesgue measure) fails to have the Kadec-Klee property. In 1936, Clarkson [4] introduced the notion of the uniform convexity property $(U C)$ or the uniform rotund property $(U R)$ of Banach spaces, and it was shown that $L_{p}$ with $1<p<\infty$ are examples of such space. In 1967, Opial [5] introduced a new property which was called the Opial property and proved that the sequence spaces $l_{p}$ $(1<p<\infty)$ have this property but $L_{p}[0, \pi](p \neq 2,1<p<\infty)$ do not have it. In 1980, Huff [6] introduced the nearly uniform convexity for Banach spaces and he also proved that every nearly uniformly convex Banach space is reflexive and it has the uniformly Kadec-Klee property (UKK). In 1987, Rolewicz [7] defined the drop property and property $(\beta)$ and the characterization of property $(\beta)$, which is proved in [8]. In 1991, Kutzarova [8] defined and studied k-nearly uniformly Banach spaces. In 1992, Prus [9] introduced the notion of the uniform Opial property. There are many papers about the geometrical properties of sequence spaces. In 2003, Suantai [10, 11] defined the generalized Cesàro sequence space with a bounded sequence $p=\left(p_{k}\right)$ of positive real numbers. In 2010, Şimşek et al. [12] introduced a new modular sequence space which is more general than the Cesàro sequence

\section{空 Springer}

๑2014 Latif et al.; licensee Springer. This is an Open Access article distributed under the terms of the Creative Commons Attribution License (http://creativecommons.org/licenses/by/2.0), which permits unrestricted use, distribution, and reproduction in any medium, provided the original work is properly cited. 
space defined by Shiue [13] and the generalized Cesàro sequence space defined by Suantai. In 2013, Mongkolkeha and Kumam [14] defined the generalized Cesàro sequence space $\operatorname{ces}_{(p)}(q)$ for a bounded sequence $p=\left(p_{k}\right)$ with $p_{k} \geq 1$ for all $k \in \mathbb{N}$ and $q=\left(q_{k}\right)$ of positive real numbers. Recently, Şimşek et al. $[15,16]$ defined it by the modular sequence space with de la Vallée-Poussin's mean and studied some geometric properties in these spaces. Some examples of the geometry of sequence spaces and their generalizations have been extensively studied in [17-20].

The main purpose of this paper is to investigate the property $(\beta)$ and the uniform Opial property equipped with the Luxemburg norm of the new modular sequence space, which is defined by using the generalized de la Vallée-Poussin mean with generalized Riesz transformation. Furthermore, we show that this space has the fixed point property.

\section{Preliminaries and notations}

Let $l^{0}$ be the space of all real sequences. For $1 \leq p<\infty$, the Cesàro sequence space ( $\operatorname{ces}_{p}$, for short) of Shue is defined by

$$
\operatorname{ces}_{p}=\left\{x \in l^{0}: \sum_{k=1}^{\infty}\left(\frac{1}{k} \sum_{i=0}^{k}|x(i)|\right)^{p}<\infty\right\}
$$

equipped with the norm

$$
\|x\|=\left(\sum_{k=1}^{\infty}\left(\frac{1}{k} \sum_{i=1}^{k}|x(i)|\right)^{p}\right)^{\frac{1}{p}} .
$$

The generalized Cesàro sequence space $\operatorname{ces}(p)$ for $p=\left(p_{k}\right)$ a bounded sequence of positive real numbers with $p_{k} \geq 1$ for all $k \in \mathbb{N}$ of Suantai $[10,11]$ is defined by

$$
\operatorname{ces}_{(p)}=\left\{x \in l^{0}: \varrho(\lambda x)<\infty \text { for some } \lambda>0\right\},
$$

where

$$
\varrho(x)=\sum_{k=1}^{\infty}\left(\frac{1}{k} \sum_{i=1}^{k}|x(i)|\right)^{p_{k}}
$$

equipped with the Luxemburg norm

$$
\|x\|=\inf \left\{\varepsilon>0: \varrho\left(\frac{x}{\varepsilon}\right) \leq 1\right\} .
$$

In the case when $p_{k}=p, 1 \leq p<\infty$ for all $k \in \mathbb{N}$, the generalized Cesàro sequence space $\operatorname{ces}_{(p)}$ is nothing but the Cesàro sequence space ces ${ }_{p}$ and the Luxemburg norm is expressed by $(2.1)$.

Let $\wedge=\left(\lambda_{k}\right)$ be a nondecreasing sequence of positive real numbers tending to infinity and let $\lambda_{1}=1$ and $\lambda_{k+1} \leq \lambda_{k}+1$. The generalized de la Vallée-Poussin means of a sequence $x=\left(x_{k}\right)$ is defined as follows:

$$
t_{k}(x)=\frac{1}{\lambda_{k}} \sum_{j \in I_{k}} x_{j} \quad \text { where } I_{k}=\left[k-\lambda_{k}+1, k\right] \text { for } k \geq 1 .
$$


The modular sequence space $V_{\varrho}(\lambda ; p)$ of Şimşek et al. $[15,16]$ is defined by de la ValléePoussin's mean, namely

$$
V_{\varrho}(\lambda ; p)=\left\{x \in l^{0}: \varrho(\tau x)<\infty \text { for some } \tau>0\right\}
$$

where

$$
\varrho(x)=\sum_{k=1}^{\infty}\left(\frac{1}{\lambda_{k}} \sum_{i \in I_{k}}|x(i)|\right)^{p_{k}}
$$

equipped with the Luxemburg norm

$$
\|x\|=\inf \left\{\tau>0: \varrho\left(\frac{x}{\tau}\right) \leq 1\right\} .
$$

Let $q=\left(q_{k}\right)$ be a sequence of positive real numbers and $Q_{k}=\sum_{i=1}^{k} q_{i}$. Then the Riesz transformation of $x=\left(x_{k}\right)$ is defined as

$$
t_{k}=\frac{1}{Q_{k}} \sum_{i=1}^{k} q_{i} x_{i}
$$

In 2012, Mursaleen et al. [21] has modified the definition of weighted statistical convergence due to Karakaya and Chishti [22], they showed that the definition must be as follows: A sequence $x=\left(x_{k}\right)$ is weighted statistically convergent (or $S_{\bar{N}}$-convergent) to $L$ if, for every $\varepsilon>0$,

$$
\lim _{k \rightarrow \infty} \frac{1}{Q_{k}}\left|\left\{i \leq Q_{k}: q_{i}\left|x_{i}-L\right| \geq \varepsilon\right\}\right|=0,
$$

where $Q_{k}=\sum_{i=1}^{k} q_{i} \rightarrow \infty$ as $k \rightarrow \infty$. In the same year, Mongkolkeha and Kumam [14]

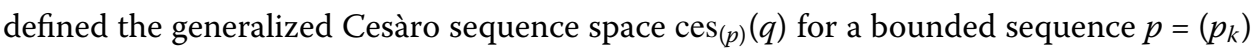
with $p_{k} \geq 1$ for all $k \in \mathbb{N}$ and $q=\left(q_{k}\right)$ of positive real numbers by

$$
\operatorname{ces}_{(p)}(q)=\left\{x \in l^{0}: \varrho(\lambda x)<\infty \text { for some } \lambda>0\right\},
$$

where

$$
\varrho(x)=\sum_{k=1}^{\infty}\left(\frac{1}{Q_{k}} \sum_{i=1}^{k} q_{i}|x(i)|\right)^{p_{k}}
$$

and $Q_{k}=\sum_{i=1}^{k} q_{i}$ with $Q_{k}=\sum_{i=1}^{k} q_{i} \rightarrow \infty$ as $k \rightarrow \infty$. Thus, we see that $p_{k}=p, 1 \leq p<$ $\infty$ for all $k \in \mathbb{N}$; then $\operatorname{ces}_{(p)}(q)$ reduces to $\operatorname{ces}_{p}(q)$ defined by Khan [23]. Recently, Belen and Mohiuddine [24] generalized the concept of weighted statistical convergence due to Mursaleen et al. for a nondecreasing sequence $\left(\lambda_{k}\right)$ of positive real numbers tending to infinity and let $\lambda_{k}=1$ and $\lambda_{k+1} \leq \lambda_{k}+1$. That is, let a sequence $q=\left(q_{k}\right)$ of nonnegative 
numbers be such that $q_{0}>0$ and $Q_{\lambda_{k}}=\sum_{i \in I_{k}} q_{i} \rightarrow \infty$ as $k \rightarrow \infty$ and

$$
\sigma_{k}:=\frac{1}{Q_{\lambda_{k}}} \sum_{i \in I_{k}} q_{i}
$$

where $I_{k}=\left[k-\lambda_{k}+1, k\right]$.

A sequence $x=\left(x_{k}\right)$ is weighted $\lambda$-statistically convergent (or $S_{\bar{N}_{\lambda}}$-convergent) to $L$ if for every $\varepsilon>0$,

$$
\lim _{n \rightarrow \infty} \frac{1}{Q_{\lambda_{k}}}\left|\left\{i \leq Q_{\lambda_{k}}: q_{i}\left|x_{i}-L\right| \geq \varepsilon\right\}\right|=0
$$

Now, we define the new generalized modular sequence space for $p=\left(p_{k}\right)$ a bounded sequence of positive real numbers with $p_{k} \geq 1$ for all $k \in \mathbb{N}$ and $\left(q_{k}\right)$ is a sequence of positive real numbers such that $q_{0}>0$. Let $Q_{\lambda_{k}}=\sum_{i \in I_{k}} q_{i} \rightarrow \infty$ as $k \rightarrow \infty$ by

$$
V_{\varrho}(\lambda ; p, q)=\left\{x \in l^{0}: \varrho(\tau x)<\infty \text { for some } \tau>0\right\},
$$

where

$$
\varrho(x)=\sum_{k=1}^{\infty}\left(\frac{1}{Q_{\lambda_{k}}} \sum_{i \in I_{k}} q_{i}|x(i)|\right)^{p_{k}}
$$

equipped with the Luxemburg norm

$$
\|x\|=\inf \left\{\tau>0: \varrho\left(\frac{x}{\tau}\right) \leq 1\right\}
$$

when $Q_{\lambda_{k}}=\sum_{i \in I_{k}} q_{i}$ and $I_{k}=\left[k-\lambda_{k}+1, k\right]$ for $k \geq 1$.

By applying the reasoning of Remark 2.4 in [24], if we take $\lambda_{k}=k$ for all $k \geq 1$, then the weighted generalized modular sequence space $V_{\varrho}(\lambda ; p, q)$ becomes the space ces ${ }_{(p)}(q)$. If we take $q_{k}=1$ for all $k \geq 1$, then the weighted generalized modular sequence space $V_{\varrho}(\lambda ; p, q)$ becomes the space $V_{\varrho}(\lambda ; p)$. Also, if we take $\lambda_{k}=k$ and $q_{k}=1$ for all $k \geq 1$, then the weighted generalized modular sequence space $V_{\varrho}(\lambda ; p, q)$ becomes the space $\operatorname{ces}_{(p)}$.

Let $(X,\|\cdot\|)$ be a real Banach space and let $B(X)$ (resp., $S(X))$ be a closed unit ball (resp., the unit sphere) of $X$. A point $x \in S(X)$ is an $H$-point of $B(X)$ if for any sequence $\left(x_{n}\right)$ in $X$ such that $\left\|x_{n}\right\| \rightarrow 1$ as $n \rightarrow \infty$, the weak convergence of $\left(x_{n}\right)$ to $x$ implies that $\| x_{n}-$ $x \| \rightarrow 0$ as $n \rightarrow \infty$. If every point in $S(X)$ is an $H$-point of $B(X)$, then $X$ is said to have the property $(H)$. A Banach space $X$ has the property $(\beta)$ if for each $\varepsilon>0$ there exists $\delta>0$ such that $1<\|x\|<1+\delta$ implies $\alpha(\operatorname{conv}(B(X) \cup\{x\}) \backslash B(X))<\varepsilon$, where $\alpha(A)$ denotes the Kuratowski measure noncompactness of a subset $A$ of $X$ defined as the infimum of all $\varepsilon>0$ such that $A$ can be covered by a finite union of sets of diameter less than $\varepsilon$. The following characterization of the property $(\beta)$ is very useful (see [25]): A Banach space $X$ has the property $(\beta)$ if and only if for each $\varepsilon>0$ there exists $\delta>0$ such that for each element $x \in B(X)$ and for each sequence $\left(x_{n}\right)$ in $B(X)$ with $\operatorname{sep}\left(x_{n}\right) \geq \varepsilon$ there is an index $k$ for which $\left\|\frac{x+x_{k}}{2}\right\|<1-\delta$ where $\operatorname{sep}\left(x_{n}\right)=\inf \left\{\left\|x_{n}-x_{m}\right\|: n \neq m\right\}>\varepsilon$. A Banach space $X$ is nearly uniformly convex (NUC) if for each $\varepsilon>0$ and every sequence $\left(x_{n}\right)$ in $B(X)$ with 
$\operatorname{sep}\left(x_{n}\right) \geq \varepsilon$, there exists $\delta \in(0,1)$ such that $\operatorname{conv}\left(x_{n}\right) \cap(1-\delta) B(X) \neq \emptyset$. A Banach space $X$ is said to have the Opial property (see [5]) if every sequence $\left(x_{n}\right)$ weakly convergent to $x_{0}$ satisfies

$$
\lim _{n \rightarrow \infty} \inf \left\|x_{n}-x_{0}\right\| \leq \lim _{n \rightarrow \infty} \inf \left\|x_{n}-x\right\|
$$

for every $x \in X$. Opial proved in [5] that the sequence spaces $l_{p}(1<p<\infty)$ have this property but $L_{p}[0, \pi](p \neq 2,1<p<\infty)$ do not have it. A Banach space $X$ is said to have the uniform Opial property (see [9]), if for each $\varepsilon>0$ there exists $\tau>0$ such that for any weakly null sequence $\left(x_{n}\right)$ in $S(X)$ and $x \in X$ with $\|x\|>\varepsilon$ the following holds:

$$
1+\tau \leq \lim _{n \rightarrow \infty} \inf \left\|x_{n}-x\right\|
$$

For example, the spaces in $[19,20]$ have the uniform Opial property.

The ball-measure of noncompactness was defined in $[26,27]$ by

$$
\beta(A)=\inf \{\varepsilon>0: A \text { can be covered by finitely many balls of diameter } \leq \varepsilon\} .
$$

A Banach space $X$ is said to have property $(L)$ if $\lim \varepsilon_{\rightarrow 1^{-}} \Delta(\varepsilon)=1$, where $\Delta(\varepsilon)=\inf \{1-$ $\inf [\|x\|: x \in A]: A$ is closed convex subset of $B(X)$ with $\beta(A) \geq \varepsilon\}$. The function $\Delta$ is called the modulus of noncompact convexity (see [26]). It has been proved in [9] that property $(L)$ is a useful tool in fixed point theory and that a Banach space $X$ has property $(L)$ if and only if it is reflexive and has the uniform Opial property.

Throughout this paper, we assume that $\lim _{k \rightarrow \infty} \inf p_{k}>1$ and $\lim _{k \rightarrow \infty} \sup p_{k}<\infty$ and for $x \in l^{0}, i \in \mathbb{N}$, we denote

$$
\begin{aligned}
& e_{i}=(\overbrace{0,0, \ldots, 0}^{i-1}, 1,0,0,0, \ldots), \\
& \left.x\right|_{i}=(x(1), x(2), x(3), \ldots, x(i), 0,0,0, \ldots), \\
& \left.x\right|_{\mathbb{N}-i}=(0,0,0, \ldots, x(i+1), x(i+2), \ldots) .
\end{aligned}
$$

In addition, we put $M=\sup _{k} p_{k}$ for all $k \geq 1$.

First, we start with a brief recollection of basic concepts and facts in modular space. For a real vector space $X$, a function $\rho: X \rightarrow[0, \infty]$ is called a modular if it satisfies the following conditions:

(i) $\rho(x)=0$ if and only if $x=0$;

(ii) $\rho(\alpha x)=\rho(x)$ for all scalar $\alpha$ with $|\alpha|=1$;

(iii) $\rho(\alpha x+\beta y) \leq \rho(x)+\rho(y)$, for all $x, y \in X$ and all $\alpha, \beta \geq 0$ with $\alpha+\beta=1$.

The modular $\rho$ is called convex if

(iv) $\rho(\alpha x+\beta y) \leq \alpha \rho(x)+\beta \rho(y)$, for all $x, y \in X$ and all $\alpha, \beta \geq 0$ with $\alpha+\beta=1$.

For modular $\rho$ on $X$, the space

$$
X_{\rho}=\left\{x \in X: \rho(\lambda x) \rightarrow 0 \text { as } \lambda \rightarrow 0^{+}\right\}
$$

is called the modular space. 
A sequence $\left(x_{n}\right)$ in $X_{\rho}$ is called modular convergent to $x \in X_{\rho}$ if there exists a $\lambda>0$ such that $\rho\left(\lambda\left(x_{n}-x\right)\right) \rightarrow 0$ as $n \rightarrow \infty$.

A modular $\rho$ is said to satisfy the $\Delta_{2}$-condition $\left(\rho \in \Delta_{2}\right)$ if for any $\varepsilon>0$ there exist constants $K \geq 2$ and $a>0$ such that

$$
\rho(2 u) \leq K \rho(u)+\varepsilon
$$

for all $u \in X_{\rho}$ with $\rho(u) \leq a$.

If $\rho$ satisfies the $\Delta_{2}$-condition for any $a>0$ with $K \geq 2$ dependent on $a$, we say that $\rho$ has the strong $\Delta_{2}$-condition $\left(\rho \in \Delta_{2}^{s}\right)$.

Lemma 2.1 [28, Lemma 2.1] If $\rho \in \Delta_{2}^{s}$, then for any $L>0$ and $\varepsilon>0$, there exists $\delta=\delta(L, \varepsilon)>$ 0 such that

$$
|\rho(u+v)-\rho(u)|<\varepsilon,
$$

whenever $u, v \in X_{\rho}$ with $\rho(u) \leq L$, and $\rho(v) \leq \delta$.

Lemma 2.2 [28, Lemma 2.3] Convergences in norm and in modular sense are equivalent in $X_{\rho}$ if $\rho \in \Delta_{2}$.

Lemma 2.3 [28, Lemma 2.4] If $\rho \in \Delta_{2}^{s}$, then for any $\varepsilon>0$ there exists $\delta=\delta(\varepsilon)>0$ such that $\|x\| \geq 1+\delta$ whenever $\rho(x) \geq 1+\varepsilon$.

\section{Main results}

In this section, we prove the property $(\beta)$ and uniform Opial property in a generalized modular sequence space $V_{\varrho}(\lambda ; p, q)$. Finally, we show that this space has the fixed point property. First we shall give some results which are very important for our consideration.

Proposition 3.1 The functional $\varrho$ is a convex modular on $V_{\varrho}(\lambda ; p, q)$.

Proof Let $x, y \in V_{\varrho}(\lambda ; p, q)$. It is obvious that $\varrho(x)=0$ if and only if $x=0$ and $\varrho(\alpha x)=\varrho(x)$ for scalar $\alpha$ with $|\alpha|=1$. Let $\alpha \geq 0, \beta \geq 0$ with $\alpha+\beta=1$. By the convexity of the function $t \mapsto|t|^{p_{k}}$, for all $k \in \mathbb{N}$, we have

$$
\begin{aligned}
\varrho(\alpha x+\beta y) & =\sum_{k=1}^{\infty}\left(\frac{1}{Q_{\lambda_{k}}} \sum_{i \in I_{k}}\left|\alpha q_{i} x(i)+\beta q_{i} y(i)\right|\right)^{p_{k}} \\
& \leq \sum_{k=1}^{\infty}\left(\alpha \frac{1}{Q_{\lambda_{k}}} \sum_{i \in I_{k}} q_{i}|x(i)|+\beta \frac{1}{Q_{\lambda_{k}}} \sum_{i \in I_{k}} q_{i}|y(i)|\right)^{p_{k}} \\
& \leq \alpha \sum_{k=1}^{\infty}\left(\frac{1}{Q_{\lambda_{k}}} \sum_{i \in I_{k}} q_{i}|x(i)|\right)^{p_{k}}+\beta \sum_{k=1}^{\infty}\left(\frac{1}{Q_{\lambda_{k}}} \sum_{i \in I_{k}} q_{i}|y(i)|\right)^{p_{k}} \\
& =\alpha \varrho(x)+\beta \varrho(y) .
\end{aligned}
$$

Proposition 3.2 For $x \in V_{\varrho}(\lambda ; p, q)$, the modular $\varrho$ on $V_{\varrho}(\lambda ; p, q)$ satisfies the following properties: 
(i) if $0<a<1$, then $a^{M} \varrho\left(\frac{x}{a}\right) \leq \varrho(x)$ and $\varrho(a x) \leq a \varrho(x)$;

(ii) if $a>1$, then $\varrho(x) \leq a^{M} \varrho\left(\frac{x}{a}\right)$;

(iii) if $a \geq 1$, then $\varrho(x) \leq a \varrho(x) \leq \varrho(a x)$.

Proof (i) Let $0<a<1$. Then we have

$$
\begin{aligned}
\varrho(x) & =\sum_{k=1}^{\infty}\left(\frac{1}{Q_{\lambda_{k}}} \sum_{i \in I_{k}} q_{i}|x(i)|\right)^{p_{k}} \\
& =\sum_{k=1}^{\infty}\left(\frac{a}{Q_{\lambda_{k}}} \sum_{i \in I_{k}} q_{i}\left|\frac{x(i)}{a}\right|\right)^{p_{k}} \\
& =\sum_{k=1}^{\infty} a^{p_{k}}\left(\frac{1}{Q_{\lambda_{k}}} \sum_{i \in I_{k}} q_{i}\left|\frac{x(i)}{a}\right|\right)^{p_{k}} \\
& \geq \sum_{k=1}^{\infty} a^{M}\left(\frac{1}{Q_{\lambda_{k}}} \sum_{i \in I_{k}} q_{i}\left|\frac{x(i)}{a}\right|\right)^{p_{k}} \\
& =a^{M} \sum_{k=1}^{\infty}\left(\frac{1}{Q_{\lambda_{k}}} \sum_{i \in I_{k}} q_{i}\left|\frac{x(i)}{a}\right|\right)^{p_{k}} \\
& =a^{M} \varrho\left(\frac{x}{a}\right) .
\end{aligned}
$$

By the convexity of modular $\varrho$, we have $\varrho(a x) \leq a \varrho(x)$, so (i) is obtained.

(ii) Let $a>1$. Then

$$
\begin{aligned}
\varrho(x) & =\sum_{k=1}^{\infty}\left(\frac{1}{Q_{\lambda_{k}}} \sum_{i \in I_{k}} q_{i}|x(i)|\right)^{p_{k}} \\
& =\sum_{k=1}^{\infty} a^{p_{k}}\left(\frac{1}{Q_{\lambda_{k}}} \sum_{i \in I_{k}} q_{i}\left|\frac{x(i)}{a}\right|\right)^{p_{k}} \\
& \leq a^{M} \sum_{k=1}^{\infty}\left(\frac{1}{Q_{\lambda_{k}}} \sum_{i \in I_{k}} q_{i}\left|\frac{x(i)}{a}\right|\right)^{p_{k}} \\
& =a^{M} \varrho\left(\frac{x}{a}\right) .
\end{aligned}
$$

Hence (ii) is satisfied. (iii) follows from the convexity of $\varrho$.

Following the line of the proof in $[10,11,17]$, we get the following results.

Proposition 3.3 For any $x \in V_{\varrho}(\lambda ; p, q)$, we have

(i) if $\|x\|<1$, then $\varrho(x) \leq\|x\|$;

(ii) if $\|x\|>1$, then $\varrho(x) \geq\|x\|$;

(iii) $\|x\|=1$ if and only if $\varrho(x)=1$;

(iv) $\|x\|<1$ if and only if $\varrho(x)<1$;

(v) $\|x\|>1$ if and only if $\varrho(x)>1$.

Proposition 3.4 For any $x \in V_{\varrho}(\lambda ; p, q)$, we have 
(i) if $0<a<1$ and $\|x\|>a$, then $\varrho(x)>a^{M}$;

(ii) if $a \geq 1$ and $\|x\|<a$, then $\varrho(x)<a^{M}$.

Proposition 3.5 Let $\left(x_{n}\right)$ be a sequence in $V_{\varrho}(\lambda ; p, q)$.

(i) If $\left\|x_{n}\right\| \rightarrow 1$ as $n \rightarrow \infty$, then $\varrho\left(x_{n}\right) \rightarrow 1$ as $n \rightarrow \infty$.

(ii) If $\varrho\left(x_{n}\right) \rightarrow 0$ as $n \rightarrow \infty$, then $\left\|x_{n}\right\| \rightarrow 0$ as $n \rightarrow \infty$.

Lemma 3.6 For any $x \in V_{\varrho}(\lambda ; p, q)$, there exist $j_{0} \in \mathbb{N}$ and $\gamma \in(0,1)$ such that $\varrho\left(\frac{x^{j}}{2}\right) \leq$ $\frac{1-\gamma}{2} \varrho\left(x^{j}\right)$ for all $j \in \mathbb{N}$ with $j \geq j_{0}$, where

$$
x^{j}=(\overbrace{0,0, \ldots, 0}^{j-1}, \Sigma_{k-\lambda_{k}+1 \leq i \leq j}|x(i)|, x(j+1), x(j+2), \ldots)
$$

and $\lambda_{k}$ corresponding to $I_{k}$ for $k \geq 1$.

Proof Let $j \in \mathbb{N}$ be fixed. So there exist $k_{j} \in \mathbb{N}$ such that $j \in I_{k_{j}}$. Let $\alpha$ be a real number such that $1<\alpha \leq \lim _{k \rightarrow \infty} \inf p_{k}$, then there exists $j_{0} \in \mathbb{N}$ such that $\alpha<p_{k_{j}}$ for all $j \geq j_{0}$. Choose $\gamma \in(0,1)$ to be a real such that $\left(\frac{1}{2}\right)^{\alpha} \leq \frac{1-\gamma}{2}$. Then for each $x \in V_{\varrho}(\lambda ; p, q)$ and $j \geq j_{0}$, we have

$$
\begin{aligned}
\varrho\left(\frac{x^{j}}{2}\right) & =\sum_{k=k_{j}}^{\infty}\left(\frac{1}{Q_{\lambda_{k}}} \sum_{i \in I_{k}} q_{i}\left|\frac{x(i)}{2}\right|\right)^{p_{k}} \\
& =\sum_{k=k_{j}}^{\infty}\left(\frac{1}{2}\right)^{p_{k}}\left(\frac{1}{Q_{\lambda_{k}}} \sum_{i \in I_{k}} q_{i}|x(i)|\right)^{p_{k}} \\
& \leq\left(\frac{1}{2}\right)^{\alpha} \sum_{k=k_{j}}^{\infty}\left(\frac{1}{Q_{\lambda_{k}}} \sum_{i \in I_{k}} q_{i}|x(i)|\right)^{p_{k}} \\
& \leq \frac{1-\gamma}{2} \varrho\left(x^{j}\right) .
\end{aligned}
$$

Lemma 3.7 For any $x \in V_{\varrho}(\lambda ; p, q)$ and $\varepsilon \in(0,1)$ there exists $\delta \in(0,1)$ such that $\varrho(x) \leq 1-\varepsilon$ implies $\|x\| \leq 1-\delta$.

Proof For a proof of this lemma, we apply and follow the line of the proof of Theorem 1.39(4) in [29]. Suppose that the lemma does not hold, then there exist $\varepsilon>0$ and $x_{n} \in$ $V_{\varrho}(\lambda ; p, q)$ such that $\varrho\left(x_{n}\right) \leq 1-\varepsilon$ and $\frac{1}{2} \leq\left\|x_{n}\right\| \nearrow 1$. Let $a_{n}=\frac{1}{\left\|x_{n}\right\|}-1$. Then $a_{n} \rightarrow 0$ as $n \rightarrow$ $\infty$. Let $L=\sup \left\{\varrho\left(2 x_{n}\right): n \in \mathbb{N}\right\}$. By $\sup _{k} p_{k}<\infty$, i.e., $\varrho \in \Delta_{2}^{s}$, there exists $K \geq 2$ such that

$$
\varrho(2 u) \leq K \varrho(u)+1,
$$

for every $u \in l(p, \theta)$ with $\varrho(u)<1$. By (3.1), we have $\varrho\left(2 x_{n}\right) \leq K \varrho\left(x_{n}\right)+1 \leq K+1$ for all $n \in \mathbb{N}$. Hence $0<L<\infty$. By Proposition 3.1 and Proposition 3.2(iii), we have

$$
\begin{aligned}
1 & =\varrho\left(\frac{x_{n}}{\left\|x_{n}\right\|}\right)=\varrho\left(2 a_{n} x_{n}+\left(1-a_{n}\right) x_{n}\right) \leq a_{n} \varrho\left(2 x_{n}\right)+\left(1-a_{n}\right) \varrho\left(x_{n}\right) \\
& \leq a_{n} L+(1-\varepsilon) \rightarrow 1-\varepsilon
\end{aligned}
$$

which is a contradiction. 
Theorem 3.8 The space $V_{\varrho}(\lambda ; p, q)$ is a Banach space with respect to the Luxemburg norm.

Proof Let $\left(x_{n}\right)=\left(x_{n}(i)\right)$ be a Cauchy sequence in $V_{\varrho}(\lambda ; p, q)$ and $\varepsilon \in(0,1)$. Thus there exists $N \in \mathbb{N}$ such that $\left\|x_{n}-x_{m}\right\|<\varepsilon$ for all $n, m \geq N$. By Proposition 3.3(i), we have

$$
\varrho\left(x_{n}-x_{m}\right) \leq\left\|x_{n}-x_{m}\right\|<\varepsilon \quad \text { for all } n, m \geq N \text {. }
$$

That is,

$$
\sum_{k=1}^{\infty}\left(\frac{1}{Q_{\lambda_{k}}} \sum_{i \in I_{k}} q_{i}\left|x_{n}(i)-x_{m}(i)\right|\right)^{p_{k}}<\varepsilon \quad \text { for all } n, m \geq N
$$

For fixed $k$, we see that

$$
\left|q_{i} x_{n}(i)-q_{i} x_{m}(i)\right|<\varepsilon \quad \text { for all } n, m \geq N
$$

Thus, let $\left(q_{i} x_{n}(i)\right)$ be a Cauchy sequence in $\mathbb{R}$ for all $i \in \mathbb{N}$. Since $\mathbb{R}$ is complete, for each $i \geq 1$, there exists $x(i) \in \mathbb{R}$ such that $q_{i} x_{m}(i) \rightarrow q_{i} x(i)$ as $m \rightarrow \infty$. Thus for fixed $k$ and for each $i \in I_{k}$, we have

$$
q_{i}\left|x_{n}(i)-x(i)\right|<\varepsilon \quad \text { as } m \rightarrow \infty \text {, for all } n \geq N
$$

This implies that

$$
\varrho\left(x_{n}-x_{m}\right) \rightarrow \varrho\left(x_{n}-x\right) \quad \text { as } m \rightarrow \infty .
$$

That is,

$$
\sum_{k=1}^{\infty}\left(\frac{1}{Q_{\lambda_{k}}} \sum_{i \in I_{k}} q_{i}\left|x_{n}(i)-x_{m}(i)\right|\right)^{p_{k}} \rightarrow \sum_{k=1}^{\infty}\left(\frac{1}{Q_{\lambda_{k}}} \sum_{i \in I_{k}} q_{i}\left|x_{n}(i)-x(i)\right|\right)^{p_{k}}
$$

as $m \rightarrow \infty$. By (3.3), we have

$$
\varrho\left(x_{n}-x\right) \leq\left\|x_{n}-x\right\|<\varepsilon \quad \text { for all } n \geq N
$$

and hence $x_{n} \rightarrow x$ as $n \rightarrow \infty$. So we have $x_{n}-x \in V_{\varrho}(\lambda ; p, q)$. Since $\left(x_{n}\right) \in V_{\varrho}(\lambda ; p, q)$ and the linearity of the sequence space $V_{\varrho}(\lambda ; p, q)$, we get $x=x_{n}-\left(x_{n}-x\right) \in V_{\varrho}(\lambda ; p, q)$. Therefore the sequence space $V_{\varrho}(\lambda ; p, q)$ is a Banach space, with respect to the Luxemburg norm, and the proof is complete.

Theorem 3.9 The space $V_{\varrho}(\lambda ; p, q)$ has property $(\beta)$.

Proof Let $\varepsilon>0$ and $\left(x_{n}\right) \subset B\left(V_{\varrho}(\lambda ; p, q)\right)$ with $\operatorname{sep}\left(x_{n}\right) \geq \varepsilon$. For each $j \in \mathbb{N}$, there exist $k_{j} \in \mathbb{N}$ such that $j \in I_{k_{j}}$. Let

$$
x_{n}^{j}=(\overbrace{0,0, \ldots, 0}^{j-1}, \Sigma_{k-\lambda_{k}+1 \leq i \leq j}\left|x_{n}(i)\right|, x_{n}(j+1), x_{n}(j+2), \ldots),
$$


where $\lambda_{k}$ corresponds to $I_{k}$ for $k \geq 1$. This is so since for each $i \in \mathbb{N},\left(x_{n}(i)\right)_{n=1}^{\infty}$ is bounded. By using the diagonal method, we see that for each $j \in \mathbb{N}$ we can find a subsequence $\left(x_{n_{l}}\right)$ of $\left(x_{n}\right)$ such that $\left(x_{n_{l}}(i)\right)$ converges for each $i \in \mathbb{N}$. Therefore, for any $j \in \mathbb{N}$ there exists an increasing sequence $\left(t_{j}\right)$ such that $\operatorname{sep}\left(\left(x_{n_{l}}^{j}\right)_{l>t_{j}}\right) \geq \varepsilon$. Hence for each $j \in \mathbb{N}$ there exists a sequence of positive integers $\left(s_{j}\right)_{j=1}^{\infty}$ with $s_{1}<s_{2}<s_{3}<\cdots$ such that $\left\|x_{s_{j}}^{j}\right\| \geq \frac{\varepsilon}{2}$ and, since $\varrho \in \Delta_{2}^{s}$, by Lemma 2.2 we may assume that there exists $\eta>0$ such that $\varrho\left(x_{s_{j}}^{j}\right) \geq \eta$ for all $j \in \mathbb{N}$, that is,

$$
\sum_{k=k_{j}}^{\infty}\left(\frac{1}{Q_{\lambda_{k}}} \sum_{i \in I_{k}} q_{i}\left|x_{s_{j}}^{j}(i)\right|\right)^{p_{k}} \geq \eta
$$

for all $j \in \mathbb{N}$. On the other hand, by Lemma 3.6, there exist $j_{0} \in \mathbb{N}$ and $\gamma \in(0,1)$ such that

$$
\varrho\left(\frac{u^{j}}{2}\right) \leq \frac{1-\gamma}{2} \varrho\left(u^{j}\right)
$$

for all $u \in V_{\varrho}(\lambda ; p, q)$ and $j \geq j_{0}$. From Lemma 3.7, there exists $\delta>0$ such that for any $y \in V_{\varrho}(\lambda ; p, q)$

$$
\varrho(y) \leq 1-\frac{\gamma \eta}{4} \quad \Longrightarrow \quad\|y\| \leq 1-\delta .
$$

Since again $\varrho \in \Delta_{2}^{s}$, by Lemma 2.1 , there exists $\delta_{0}$ such that

$$
|\varrho(u+v)-\varrho(u)|<\frac{\gamma \eta}{4},
$$

whenever $\varrho(u) \leq 1$ and $\varrho(v) \leq \delta_{0}$. Since $x \in B\left(V_{\varrho}(\lambda ; p, q)\right)$, we have $\varrho(x) \leq 1$. Then there exists $j \geq j_{0}$ such that $\varrho\left(x^{j}\right) \leq \delta_{0}$. We put $u=x_{s_{j}}^{j}$ and $v=x^{j}$,

$$
\begin{aligned}
& \varrho\left(\frac{u}{2}\right)=\sum_{k=k_{j}}^{\infty}\left(\frac{1}{Q_{\lambda_{k}}} \sum_{i \in I_{k}} q_{i}\left|\frac{x_{x_{j}}(i)}{2}\right|\right)^{p_{k}}<1 \text { and } \\
& \varrho\left(\frac{v}{2}\right)=\sum_{k=k_{j}}^{\infty}\left(\frac{1}{Q_{\lambda_{k}}} \sum_{i \in I_{k}} q_{i}\left|\frac{x(i)}{2}\right|\right)^{p_{k}}<\delta_{0} .
\end{aligned}
$$

From (3.7) and (3.9), we have

$$
\begin{aligned}
\sum_{k=k_{k}}^{\infty}\left(\frac{1}{Q_{\lambda_{k}}} \sum_{i \in I_{k}} q_{i}\left|\frac{x(i)+x_{s_{j}}(i)}{2}\right|\right)^{p_{k}} & =\varrho\left(\frac{u+v}{2}\right) \leq \varrho\left(\frac{u}{2}\right)+\frac{\gamma \eta}{4} \\
& \leq \frac{1-\gamma}{2}(\varrho(u))+\frac{\gamma \eta}{4} .
\end{aligned}
$$

By (3.6), (3.9), (3.10), and convexity of the function $f(t)=|t|^{p_{k}}$, for all $k \in \mathbb{N}$, we have

$$
\begin{aligned}
\varrho\left(\frac{x+x_{s_{j}}}{2}\right) & =\sum_{k=1}^{\infty}\left(\frac{1}{Q_{\lambda_{k}}} \sum_{i \in I_{k}} q_{i}\left|\frac{x(i)+x_{s_{j}}(i)}{2}\right|\right)^{p_{k}} \\
& =\sum_{k=1}^{k_{j}-1}\left(\frac{1}{Q_{\lambda_{k}}} \sum_{i \in I_{k}} q_{i}\left|\frac{x(i)+x_{s_{j}}(i)}{2}\right|\right)^{p_{k}}+\sum_{k=k_{j}}^{\infty}\left(\frac{1}{Q_{\lambda_{k}}} \sum_{i \in I_{k}} q_{i}\left|\frac{x(i)+x_{s_{j}}(i)}{2}\right|\right)^{p_{k}}
\end{aligned}
$$




$$
\begin{aligned}
\leq & \frac{1}{2}\left(\sum_{k=1}^{k_{j}-1}\left(\frac{1}{Q_{\lambda_{k}}} \sum_{i \in I_{k}} q_{i}|x(i)|\right)^{p_{k}}+\sum_{k=1}^{k_{j}-1}\left(\frac{1}{Q_{\lambda_{k}}} \sum_{i \in I_{k}} q_{i}\left|x_{s_{j}}(i)\right|\right)^{p_{k}}\right) \\
& +\sum_{k=k_{j}}^{\infty}\left(\frac{1}{Q_{\lambda_{k}}} \sum_{i \in I_{k}} q_{i}\left|\frac{x_{s_{j}}(i)}{2}\right|\right)^{p_{k}}+\frac{\gamma \eta}{4} \\
\leq & \frac{1}{2}\left(\sum_{k=1}^{k_{j}-1}\left(\frac{1}{Q_{\lambda_{k}}} \sum_{i \in I_{k}} q_{i}|x(i)|\right)^{p_{k}}+\sum_{k=1}^{k_{j}-1}\left(\frac{1}{Q_{\lambda_{k}}} \sum_{i \in I_{k}} q_{i}\left|x_{s_{j}}(i)\right|\right)^{p_{j}}\right) \\
& +\frac{1-\gamma}{2} \sum_{k=k_{j}}^{\infty}\left(\frac{1}{Q_{\lambda_{k}}} \sum_{i \in I_{k}} q_{i}\left|x_{s_{j}}(i)\right|\right)^{p_{k}}+\frac{\gamma \eta}{4} \\
= & \frac{1}{2} \sum_{k=1}^{k_{j}-1}\left(\frac{1}{Q_{\lambda_{k}}} \sum_{i \in I_{k}} q_{i}|x(i)|\right)^{p_{k}}+\frac{1}{2} \sum_{k=1}^{k_{j}-1}\left(\frac{1}{Q_{\lambda_{k}}} \sum_{i \in I_{k}} q_{i}\left|x_{s_{j}}(i)\right|\right)^{p_{k}} \\
& +\frac{1-\gamma}{2} \sum_{k=k_{j}}^{\infty}\left(\frac{1}{Q_{\lambda_{k}}} \sum_{i \in I_{k}} q_{i}\left|x_{s_{j}}(i)\right|\right)^{p_{k}}+\frac{\gamma \eta}{4} \\
= & \frac{1}{2} \sum_{k=1}^{k_{j}-1}\left(\frac{1}{Q_{\lambda_{n}}} \sum_{i \in I_{n}} q_{i}|x(i)|\right)^{p_{k}}+\frac{1}{2} \sum_{k=1}^{\infty}\left(\frac{1}{Q_{\lambda_{k}}} \sum_{i \in I_{k}} q_{i}\left|x_{s_{j}}(i)\right|\right)^{p_{k}} \\
& -\frac{\gamma}{2} \sum_{k=k_{j}}^{\infty}\left(\frac{1}{Q_{\lambda_{k}}} \sum_{i \in I_{k}} q_{i}\left|x_{s_{j}}(i)\right|\right)^{p_{j}}+\frac{\gamma \eta}{4} \\
= & \frac{1}{2}-\frac{\gamma \eta}{2}+\frac{\gamma \eta}{4} . \\
& \frac{\gamma \eta}{4} .
\end{aligned}
$$

So it follows from (3.8) that

$$
\left\|\frac{x+x_{s_{j}}}{2}\right\| \leq 1-\delta
$$

Therefore, the space $V_{\varrho}(\lambda ; p, q)$ has property $(\beta)$.

By the facts that property $(\beta)$ implies (NUC), and $(N U C)$ implies property $(U K K)$, property $(H)$, and reflexivity (see [29-31]). The following results are obtained directly from Theorem 3.9.

Corollary 3.10 The space $V_{\varrho}(\lambda ; p)$ has property $(\beta)$.

Corollary 3.11 The space $V_{\varrho}(\lambda ; p, q)$ is nearly uniform convexity and reflexive.

Corollary 3.12 The space $V_{\varrho}(\lambda ; p, q)$ has property $(U K K)$ and property $(H)$.

Corollary 3.13 The space $V_{\varrho}(\lambda ; p)$ is nearly uniform convexity and reflexive.

Corollary 3.14 The space $V_{\varrho}(\lambda ; p)$ has property $(U K K)$ and $(H)$. 
Next, we will prove the uniform Opial property for the space $V_{\varrho}(\lambda ; p, q)$.

Theorem 3.15 The space $V_{\varrho}(\lambda ; p, q)$ has the uniform Opial property.

Proof Take any $\varepsilon>0$ and $x \in V_{\varrho}(\lambda ; p, q)$ with $\|x\| \geq \varepsilon$. Let $\left(x_{n}\right)$ be a weakly null sequence in $S\left(V_{\varrho}(\lambda ; p, q)\right)$. By $\varrho \in \Delta_{2}^{s}$, hence by Lemma 2.2 there exists $\delta \in(0,1)$ independent of $x$ such that $\varrho(x)>\delta$. Also, by $\varrho \in \Delta_{2}^{s}$ and Lemma 2.1, one may assert that there exists $\delta_{1} \in(0, \delta)$ such that

$$
|\varrho(y+z)-\varrho(y)|<\frac{\delta}{4}
$$

whenever $\varrho(y) \leq 1$ and $\varrho(z) \leq \delta_{1}$. Choose $k_{0} \in \mathbb{N}$ such that

$$
\sum_{k=k_{0}+1}^{\infty}\left(\frac{1}{Q_{\lambda_{k}}} \sum_{i \in I_{k}} q_{i}|x(i)|\right)^{p_{k}}<\frac{\delta_{1}}{4}
$$

So, we have

$$
\begin{aligned}
\delta & <\sum_{k=1}^{k_{0}}\left(\frac{1}{Q_{\lambda_{k}}} \sum_{i \in I_{k}} q_{i}|x(i)|\right)^{p_{k}}+\sum_{k=k_{0}+1}^{\infty}\left(\frac{1}{Q_{\lambda_{k}}} \sum_{i \in I_{k}} q_{i}|x(i)|\right)^{p_{k}} \\
& \leq \sum_{k=1}^{k_{0}}\left(\frac{1}{Q_{\lambda_{k}}} \sum_{i \in I_{k}} q_{i}|x(i)|\right)^{p_{k}}+\frac{\delta_{1}}{4},
\end{aligned}
$$

which implies that

$$
\begin{aligned}
\sum_{k=1}^{k_{0}}\left(\frac{1}{\left.Q_{\lambda_{k}} \sum_{i \in I_{k}} q_{i}|x(i)|\right)^{p_{k}}}\right. & >\delta-\frac{\delta_{1}}{4} \\
& >\delta-\frac{\delta}{4} \\
& =\frac{3 \delta}{4} .
\end{aligned}
$$

Since $x_{n} \stackrel{w}{\rightarrow} 0$, there exists $n_{0} \in \mathbb{N}$ such that

$$
\frac{3 \delta}{4} \leq \sum_{k=1}^{k_{0}}\left(\frac{1}{Q_{\lambda_{k}}} \sum_{i \in I_{k}} q_{i}\left|x_{n}(i)+x(i)\right|\right)^{p_{k}}
$$

for all $n>n_{0}$, since weak convergence implies coordinatewise convergence. Again, by $x_{n} \stackrel{w}{\rightarrow} 0$, there exists $n_{1} \in \mathbb{N}$ such that

$$
\left\|x_{\left.n\right|_{k_{o}}}\right\|<1-\left(1-\frac{\delta}{4}\right)^{\frac{1}{M}}
$$

for all $n>n_{1}$. Hence, by the triangle inequality of the norm, we get

$$
\left\|x_{\left.n\right|_{\mathbb{N}-k_{o}}}\right\|>\left(1-\frac{\delta}{4}\right)^{\frac{1}{M}}
$$


It follows from Proposition 3.3(ii) that

$$
\begin{aligned}
1 & \leq \varrho\left(\frac{x_{n \mid \mathbb{N}-k_{o}}}{\left(1-\frac{\delta}{4}\right)^{\frac{1}{M}}}\right) \\
& =\sum_{k=k_{0}+1}^{\infty}\left(\frac{\frac{1}{Q_{\lambda_{k}}} \sum_{i \in I_{k}} q_{i}\left|x_{n}(i)\right|}{\left(1-\frac{\delta}{4}\right)^{\frac{1}{M}}}\right)^{p_{k}} \\
& \leq\left(\frac{1}{\left(1-\frac{\delta}{4}\right)^{\frac{1}{M}}}\right)^{M} \sum_{k=k_{0}+1}^{\infty}\left(\frac{1}{Q_{\lambda_{k}}} \sum_{i \in I_{k}} q_{i}\left|x_{n}(i)\right|\right)^{p_{k}}
\end{aligned}
$$

implies that

$$
\sum_{k=k_{0}+1}^{\infty}\left(\frac{1}{Q_{\lambda_{k}}} \sum_{i \in I_{k}} q_{i}\left|x_{n}(i)\right|\right)^{p_{k}} \geq 1-\frac{\delta}{4}
$$

for all $n>n_{1}$. By inequality (3.11), (3.12), (3.15), and (3.19), we have for any $n>n_{1}$

$$
\begin{aligned}
\varrho\left(x_{n}+x\right) & =\sum_{k=1}^{k_{0}}\left(\frac{1}{Q_{\lambda_{k}}} \sum_{i \in I_{k}} q_{i}\left|x_{n}(i)+x(i)\right|\right)^{p_{k}}+\sum_{k=k_{0}+1}^{\infty}\left(\frac{1}{Q_{\lambda_{k}}} \sum_{i \in I_{k}} q_{i}\left|x_{n}(i)+x(i)\right|\right)^{p_{k}} \\
& >\sum_{k=1}^{k_{0}}\left(\frac{1}{Q_{\lambda_{k}}} \sum_{i \in I_{k}} q_{i}\left|x_{n}(i)+x(i)\right|\right)^{p_{k}}+\sum_{k=k_{0}+1}^{\infty}\left(\frac{1}{Q_{\lambda_{k}}} \sum_{i \in I_{k}} q_{i}\left|x_{n}(i)+x(i)\right|\right)^{p_{k}} \\
& \geq \frac{3 \delta}{4}+\sum_{k=k_{0}+1}^{\infty}\left(\frac{1}{Q_{\lambda_{k}}} \sum_{i \in I_{k}} q_{i}\left|x_{n}(i)\right|\right)^{p_{k}}-\frac{\delta}{4} \\
& \geq \frac{3 \delta}{4}+\left(1-\frac{\delta}{4}\right)-\frac{\delta}{4} \\
& \geq 1+\frac{\delta}{4} .
\end{aligned}
$$

Since $\varrho \in \Delta_{2}^{s}$ and by Lemma 2.3 there exists $\tau$ depending on $\delta$ only such that $\left\|x_{n}+x\right\| \geq$ $1+\tau$, which implies that $\lim _{n \rightarrow \infty} \inf \left\|x_{n}+x\right\| \geq 1+\tau$, hence the proof is complete.

Corollary 3.16 The space $V_{\varrho}(\lambda ; p)$ has the uniform Opial property.

Corollary 3.17 [20, Theorem 2.6] The space $\operatorname{ces}_{(p)}$ has the uniform Opial property.

Corollary 3.18 [19, Theorem 2] For any $1<p<\infty$, the space ces chas the uniform Opial property.

Corollary 3.19 The space $V_{\varrho}(\lambda ; p, q)$ has property $(L)$ and the fixed point property.

Corollary 3.20 The space $V_{\varrho}(\lambda ; p)$ has property $(L)$ and the fixed point property. 
Authors' contributions

All authors contributed equally and significantly in writing this paper. All authors read and approved the final manuscript.

\section{Author details}

'Department of Mathematics, King Abdulaziz University, P.O. Box 80203, Jeddah, 21589, Saudi Arabia. ${ }^{2}$ Department of Mathematics, Faculty of Liberal Arts and Science, Kasetsart University, Kamphaeng-Saen Campus, Nakhonpathom, 73140, Thailand. ${ }^{3}$ Department of Mathematics and Statistics, Faculty of Science and Technology, Thammasat University, Rangsit Center, Pathumthani, 12121, Thailand.

\section{Acknowledgements}

This article was funded by the Deanship of Scientific Research (DSR), King Abdulaziz University, Jeddah. The authors, therefore, acknowledge with thanks DSR for the technical and financial support.

Received: 24 June 2014 Accepted: 8 September 2014 Published: 29 Sep 2014

\section{References}

1. Radon, J: Theorie und Anwendungen der absolut additiven Mengenfunctionen. Sitzungsber. Akad. Wiss. Wien 122, 1295-1438 (1913)

2. Riesz, F: Sur la convergence en moyenne I. Acta Sci. Math. 4, 58-64 (1928/1929)

3. Riesz, F: Sur la convergence en moyenne II. Acta Sci. Math. 4, 182-185 (1928/1929)

4. Clarkson, JA: Uniformly convex spaces. Trans. Am. Math. Soc. 40, 396-414 (1936)

5. Opial, Z: Weak convergence of the sequence of successive approximations for non expensive mappings. Bull. Am. Math. Soc. 73, 591-597 (1967)

6. Huff, R: Banach spaces which are nearly uniformly convex. Rocky Mt. J. Math. 10,473-479 (1980)

7. Rolewicz, S: On $\Delta$-uniform convexity and drop property. Stud. Math. 87, 181-191 (1987)

8. Kutzarova, D: k- $\beta$ and k-nearly uniformly convex Banach spaces. J. Math. Anal. Appl. 162(2), 322-338 (1991)

9. Prus, S: Banach spaces with uniform Opial property. Nonlinear Anal., Theory Methods Appl. 18(8), $697-704$ (1992)

10. Suantai, S: On H-property of some Banach sequence spaces. Arch. Math. 39, 309-316 (2003)

11. Suantai, S: On some convexity properties of generalized Cesàro sequence spaces. Georgian Math. J. 10, 193-200 (2003)

12. Şimşek, N, Savaş, E, Karakaya, V: Some geometric and topological properties of a new sequence space defined by de la Vallée-Poussin mean. J. Comput. Anal. Appl. 12(4), 768-779 (2010)

13. Shiue, JS: Cesàro sequence spaces. Tamkang J. Math. 1, 143-150 (1970)

14. Mongkolkeha, C, Kumam, P: On H-properties and uniform Opial property of generalized Cesaro sequence spaces. J. Inequal. Appl. 2012, Article ID 76 (2012)

15. Şimşek, N, Savaş, E, Karakaya, V: On geometrical properties of some Banach spaces. Appl. Math. Inf. Sci. 7(1), 295-300 (2013)

16. Şimşek, N, Savaş, E, Karakaya, V: Some geometric and topological properties of a new sequence space defined by de la Vallée-Poussin mean. J. Comput. Anal. Appl. 12(4), 768-779 (2010)

17. Mongkolkeha, C, Kumam, P: Some geometric properties of lacunary sequence spaces related to fixed point property. Abstr. Appl. Anal. 2011, Article ID 903736 (2011)

18. Sanhan, W, Suantai, S: Some geometric properties of Cesàro sequence space. Kyungpook Math. J. 43(2), 191-197 (2003)

19. Cui, Y, Hudzik, H: Some geometric properties related to fixed point theory in Cesàro space. Collect. Math. 50(3), 277-288 (1999)

20. Petrot, N, Suantai, S: Uniform Opial properties in generalized Cesàro sequence spaces. Nonlinear Anal. 63, 1116-1125 (2005)

21. Mursaleen, M, Karakaya, V, Ertürk, M, Gürsoy, F: Weighted statistical convergence and its application to Korovkin type approximation theorem. Appl. Math. Comput. 218, 9132-9137 (2012)

22. Karakaya, V, Chishti, T: Weighted statistical convergence. Iran. J. Sci. Technol., Trans. A, Sci. 33, $219-223$ (2009)

23. Khan, VA: Some geometric properties of generalized Cesàro sequence spaces. Acta Math. Univ. Comen. 1, 1-8 (2010)

24. Belen, C, Mohiuddine, SA: Generalized weighted statistical convergence and application. Appl. Math. Comput. 219, 982-9826 (2013)

25. Kutzarowa, DN: An isomorphic characterization of property $(\beta)$ of Rolwewicz. Note Mat. 10(2), 347-354 (1990)

26. Goebel, R, Kirk, WA: Topics in Metric Fixed Point Theory. Cambridge University press, Cambridge (1990)

27. Kirk, WA: A fixed point theorem for mappings which do not increase distances. Am. Math. Mon. 72, 1004-1006 (1965)

28. Cui, Y, Hudzik, H: On the uniform Opial property in some modular sequence spaces. Funct. Approx. Comment. Math. 26, 93-102 (1998)

29. Chen, S: Dissertationes mathematicae (Rozprawy Matematyczne). Instytut Matematyczny PAN, Warszawa (1996)

30. Huff, R: Bancah spaces which are near uniformly convex. Rocky Mt. J. Math. 10, 743-749 (1980)

31. Rolewicz, S: On $\triangle$-uniform convexity and drop property. Stud. Math. 87, 181-191 (1987)

10.1186/1029-242X-2014-375

Cite this article as: Latif et al.: Some geometric properties of generalized modular sequence space derived by the generalized de la Vallée-Poussin mean. Journal of Inequalities and Applications 2014, 2014:375 